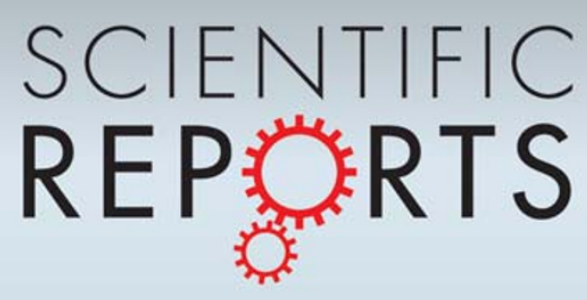

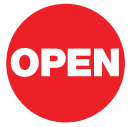

SUBJECT AREAS:

LASERS

OPTICAL MATERIALS AND STRUCTURES

SPECTROSCOPY

LIGHT SOURCES

Received

31 October 2011

Accepted

13 January 2012

Published

1 February 2012

Correspondence and requests for materials should be addressed to

H.D.S. (hdsun@ntu. edu.sg)

\section{Single Mode Lasing from Hybrid Hemispherical Microresonators}

\author{
Rui Chen, Van Duong Ta \& Han Dong Sun
}

Division of Physics and Applied Physics, School of Physical and Mathematical Sciences, Nanyang Technological University, Singapore 637371, Singapore.

Enormous attention has been paid to optical microresonators which hold a great promise for microlasers as well as fundamental studies in cavity quantum electrodynamics. Here we demonstrate a three-dimensional (3D) hybrid microresonator combining self-assembled hemispherical structure with a planar reflector. By incorporating dye molecules into the hemisphere, optically pumped lasing phenomenon is observed at room temperature. We have studied the lasing behaviors with different cavity sizes, and particularly single longitudinal mode lasing from hemispheres with diameter $\sim 15 \mu \mathrm{m}$ is achieved. Detailed characterizations indicate that the lasing modes shift under varying pump densities, which can be well-explained by frequency shift and mode hopping. This work provides a versatile approach for $3 \mathrm{D}$ confined microresonators and opens an opportunity to realize tunable single mode microlasers.

$\mathrm{n}$ recent years, the development of optical microresonators have attracted extensive research interest ${ }^{1-5}$, not only because of their small mode volume and high quality $(Q)$ factor for fundamental physics investigation ${ }^{6,7}$, but also for potential applications in such as laser sources, active filters and photonic sensing devices for labelfree detection ${ }^{8,9}$. In order to create optical microresonators, different kinds of structures and fabrication techniques have been adopted, and laser emissions have been observed from various geometries, such as Fabry-Pérot cavity $^{10,11}$, photonic crystal $^{12,13}$, random cavity ${ }^{14,15}$, plasmonic cavity ${ }^{16,17}$, distributed feedback resonator ${ }^{18}$, as well as whispering gallery modes (WGM) ${ }^{8,19}$. Among these alternatives, WGM lasing is an interesting case because of its intrinsically high $Q$ factor, low lasing threshold and relatively simple fabrication ${ }^{20-22}$.

A conventional structure which can achieve WGM is the spherical resonator. By embedding gain materials into such cavity, the emitted light can be trapped inside the sphere by multiple total internal reflections at the interface near the sphere and the surrounding medium ${ }^{23}$. More interestingly, once the confinement of light on the scale of photon wavelength is achieved, cavity quantum electrodynamics effect will take place, which will largely enhance the corresponding coupling strength ${ }^{6,24}$. As is well-known, microdroplets tend to form an almost perfect spherical shape with a smooth surface. In fact, laser emission has been demonstrated from liquid microdroplets levitated in the air by electrodynamic endcap trap ${ }^{25,26}$. However, a levitated microresonator is difficult for practical applications. A more reliable approach is the stationary microdroplets located on a super hydrophobic surface ${ }^{27,28}$. Although lasing action has been observed from such kind of structure, the microdroplets were not stable due to water evaporation. Therefore, the optical stability of the solid-state configurations under excitation condition is required for the lasing operation. Moreover, single mode lasing has not been observed to date, which limits their practical applications in laser spectroscopy and laser metrology ${ }^{29}$.

In this paper, we report the hybrid microresonators which combine self-assembled hemispherical structures with a planar reflector (either a distributed Bragg reflector or a metal mirror). Optically pumped lasing action is observed at room temperature from the dye-doped hemispheres by using a micro-photoluminescence ( $\mu$-PL) system. The analysis of the sharp peaks supports the WGM lasing mechanism. Especially, single mode lasing is obtained from hemispheres with diameter $\sim 15 \mu \mathrm{m}$. Lasing mode shift under varying pump densities is observed, which is attributed to frequency shift and mode hopping. Our studies provide a versatile approach for three dimensional (3D) confined resonators with tunable sizes.

\section{Results}

Hemispherical microresonators fabrication. The hemispherical microresonators were self-assembled through hydrophobic effect and surface tension force. As shown schematically in Figure 1, a DBR was firstly fabricated on a glass substrate by electron beam evaporation under high vacuum. The inorganic DBR comprises 27 alternating $\mathrm{TiO}_{2}$ and $\mathrm{SiO}_{2}$ quarter-wave layers for high reflectance. The reflectivity is up to $99.5 \%$ at $630 \mathrm{~nm}$ with a stop-band 


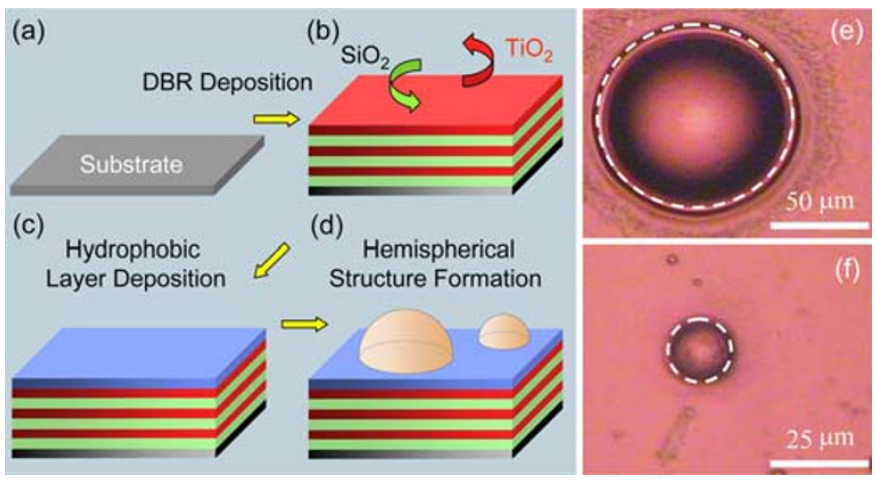

Figure $1 \mid$ Hemispherical structure fabrication. (a)-(d) Schematic diagram of the fabrication process of hemispherical structure through hydrophobic effect. (e) and (f) The optical image of the created hemispheres with halogen illumination.

width more than $140 \mathrm{~nm}$. A hydrophobic surface which is transparent to excitation light was prepared by spin coating a layer of $1 \mathrm{H}, 1 \mathrm{H}, 2 \mathrm{H}, 2 \mathrm{H}$-perfluorooctyltriethoxysilane at room temperature $^{28}$. The material used to form the hemisphere is Araldite ${ }^{\circledR} 506$ epoxy resin with high viscosity, which is purchased from SigmaAldrich. The epoxy resin was mixed with chloroform with a ratio of $8: 1$, and the dye molecules Rhodamine $6 \mathrm{G}$ were added as gain material. A drop of solution was then deposited on the hydrophobic DBR and spread by a soft felt ${ }^{30}$. By drying in air for 1 day and additionally in an oven at $80^{\circ} \mathrm{C}$ for 1 hour, solid state hemispherical microresonators with diameters ranging from 5 to $120 \mu \mathrm{m}$ can be obtained, as shown in Figure 1d. The contact angle of the hemispherical microresonators on hydrophobic DBR was determined to be $75^{\circ}$ (Figure S1 and Figure S2, Supplementary Information).

The optical images of the top-down view of hemispherical microresonators under halogen illumination are shown in Figure 1e and 1f, respectively. The background color of the image is due to the reflection of the DBR substrate. It can be seen clearly that the asfabricated structures possess a hemispherical shape and the trace lines of the structure boundaries are perfectly circular. For the hemispheres shown in Figure 1e and 1f, the diameters are estimated to be 100 and $15 \mu \mathrm{m}$, respectively.

Continuous wave laser excitation. We investigate the optical property of individual hemispheres by means of a $\mu$-PL system (see Methods for details). Figure 2a depicts the room temperature $\mu$-PL spectrum from a single hemisphere (Figure 1e, with diameter of $100 \mu \mathrm{m}$ ) excited by CW laser line of $325 \mathrm{~nm}$. As shown in Figure 2a, for pump power increasing by one order of magnitude, only one broad emission between 520 and $680 \mathrm{~nm}$ can be observed. The integrated PL intensity of this peak under different pump power is shown in Figure 2b. It is known that the PL intensity $\left(I_{P L}\right)$ can be described as a function of the laser pump power $\left(I_{E X}\right)$ by $I_{P L} \propto I_{E X}^{\alpha}$, where $\alpha$ denotes the nonlinear component ${ }^{31}$. It can be seen that the integrated PL intensity of the hemisphere increases approximately linearly with the pump power. By fitting the experimental data, a factor of $\sim 1.1$ for nonlinear component $\alpha$ can be obtained, which affirms the spontaneous emission from the dye-doped hemisphere.

WGM lasing from hemispherical microresonators. We have also examined the emission characteristics of the same hemisphere under high power density excitation through a green pulsed laser. Figure 3a shows the $\mu$-PL spectra of the hemisphere under different pump pulse energy at room temperature. It is noted that the sample displays a weak and broad spontaneous emission under low pump pulse energy such as $1.00 \mu \mathrm{J}$. As the pump pulse energy increases, multiple sharp peaks with a line-width as narrow as $\sim 0.10 \mathrm{~nm}$ emerge from the broad spontaneous peak, the intensity of which
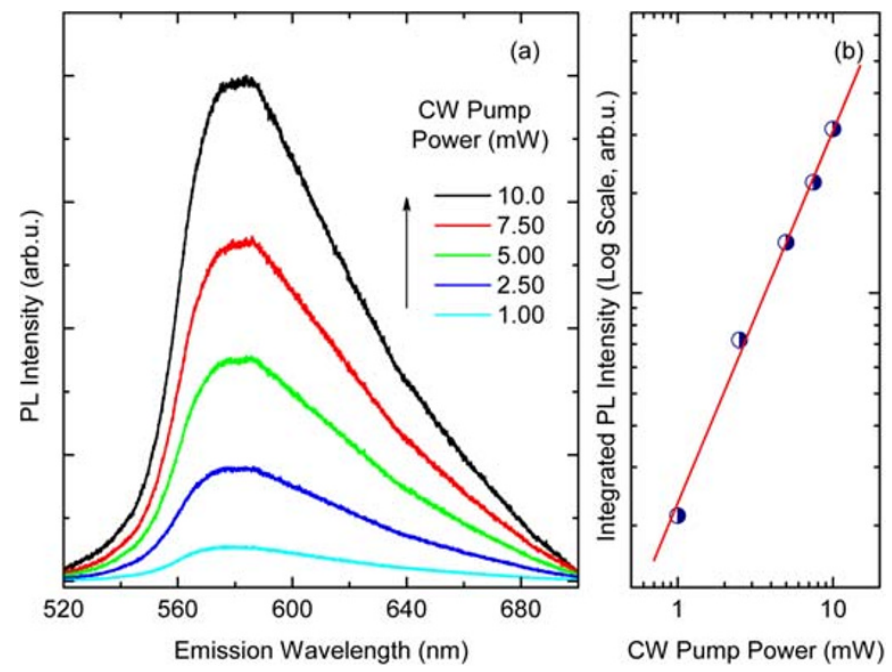

Figure $2 \mid \mathrm{CW}$ laser excitation of hemispherical microresonator. (a) The PL emission recorded at room temperature as a function of CW laser pump power. (b) The integrated PL intensity with the change of pump power.
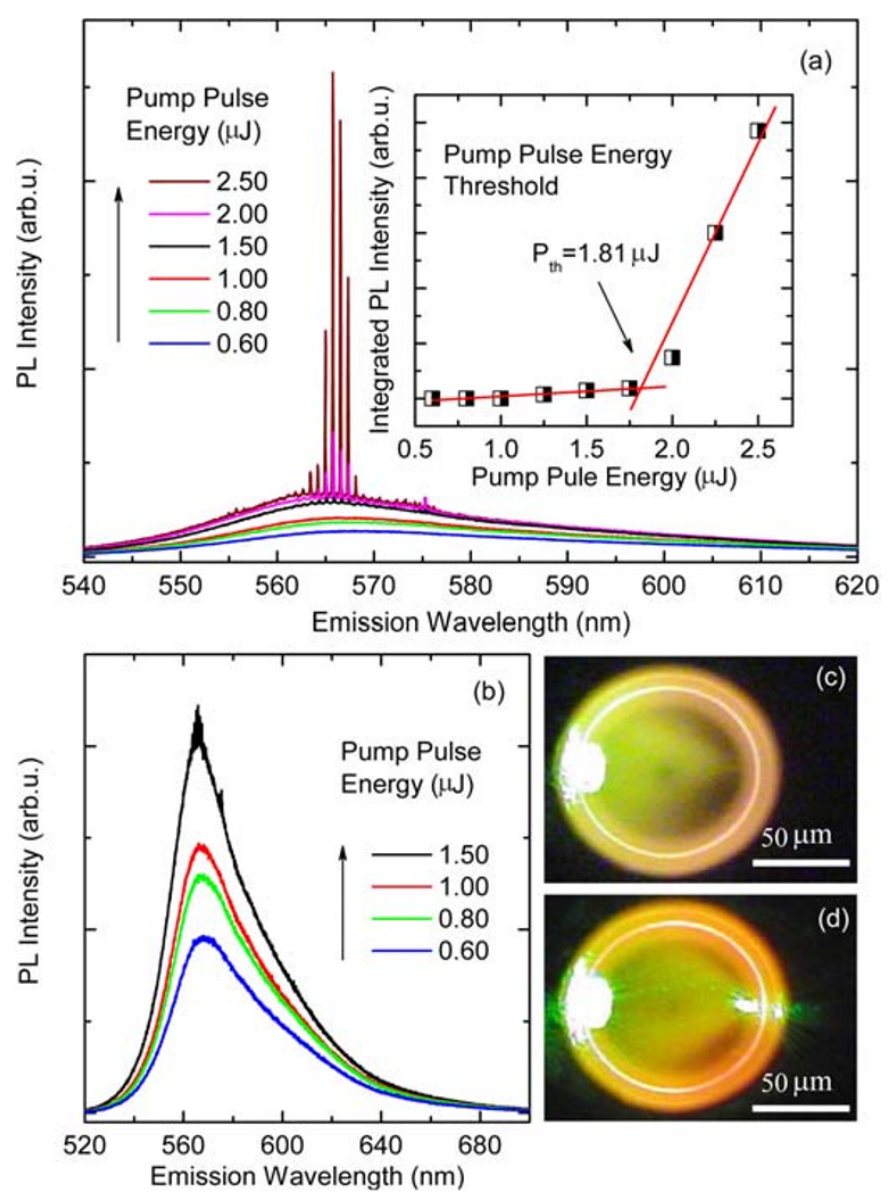

Figure 3 WGM lasing from hemispherical microresonator. (a) The $\mu$ PL spectrum of an individual hemisphere under different pump pulse energy. The inset shows the dependence of integrated PL intensity on the pump pulse energy. (b) The enlarged part of the $\mu$-PL spectrum near threshold. (c) and (d) The PL images of the hemisphere below and above threshold, respectively. 


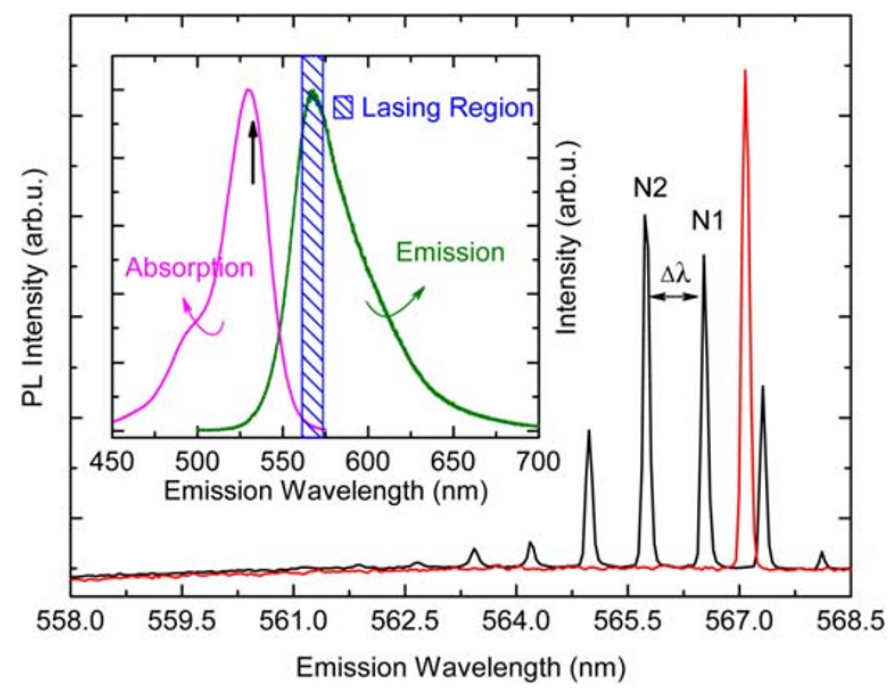

Figure 4 | Single longitudinal mode lasing. Spectrum of multimode (black curve) and single-mode lasing (red curve) from hemisphere with diameter of 100 and $15 \mu \mathrm{m}$, respectively. Inset shows the absorption and emission spectra for the dye molecules. Black arrow denotes the pump wavelength, and the blue area marks the optical gain region of the dye molecules.

increases rapidly with the further increasing of the pump pulse energy. A plot of the integrated PL intensity of these stimulated peaks with respect to the pump pulse energy is shown in the inset of Figure 3a. The observed nonlinear increase of the emission intensity is an indicator of lasing phenomenon from the hemisphere, and the lasing threshold is determined to be $1.81 \mu \mathrm{J}$. It is known that the $Q$ factor is an important parameter to describe a laser cavity. From the experiment, the $Q$ factor is estimated to be as high as $\sim 5500$ according to the definition $Q=\lambda / \delta \lambda$, where $\lambda$ is the peak wavelength and $\delta \lambda$ is the line-width of the peak, respectively. It is noted that the $Q$ factor of the proposed structure is higher than the previous reported data ${ }^{27,28}$, which may due to the perfect hemispherical surface as well as the introduction of the bottom DBR substrate.

The $\mu$-PL spectra around the threshold are magnified and plotted in Figure $3 \mathrm{~b}$. The evolution from spontaneous to stimulated emission can be seen very clearly. Moreover, the PL images of the hemisphere below and above the threshold are shown in Figure $3 \mathrm{c}$ and $3 \mathrm{~d}$, respectively. Once again, a perfect hemispherical shape of the structure can be observed clearly. A closer look at the PL images reveals the different brightness and color for the two situations. It is known that the hemisphere below the threshold demonstrates a broad band emission. However, when the optical pumping exceeds the threshold, the color of the PL image changes from peach to orange, which is due to the lasing emission at $\sim 566 \mathrm{~nm}$.

To further investigate the lasing mechanism observed from the hemisphere clearly, $\mu$-PL spectrum recorded under pump pulse energy of $2.50 \mu \mathrm{J}$ has been enlarged and presented in Figure 4 . It is found that the mode spacing of the lasing denoted as $\Delta \lambda$ is measured to be $0.79 \mathrm{~nm}$. Let $D$ and $n$ be the diameter and the effective refractive index of a circular cavity, respectively, the resonant condition can be approximately written as $\pi D n=N \lambda$, where $N$ is the mode number and $\lambda$ is resonant wavelength. For an ideal circular resonator formed by epoxy resin $(n=1.41$ and $D=95 \mu \mathrm{m})$, the mode number of the peaks N1 and N2 can be indexed as 743 and 744, respectively. The calculated mode spacing is $0.76 \mathrm{~nm}$, which agrees well with the
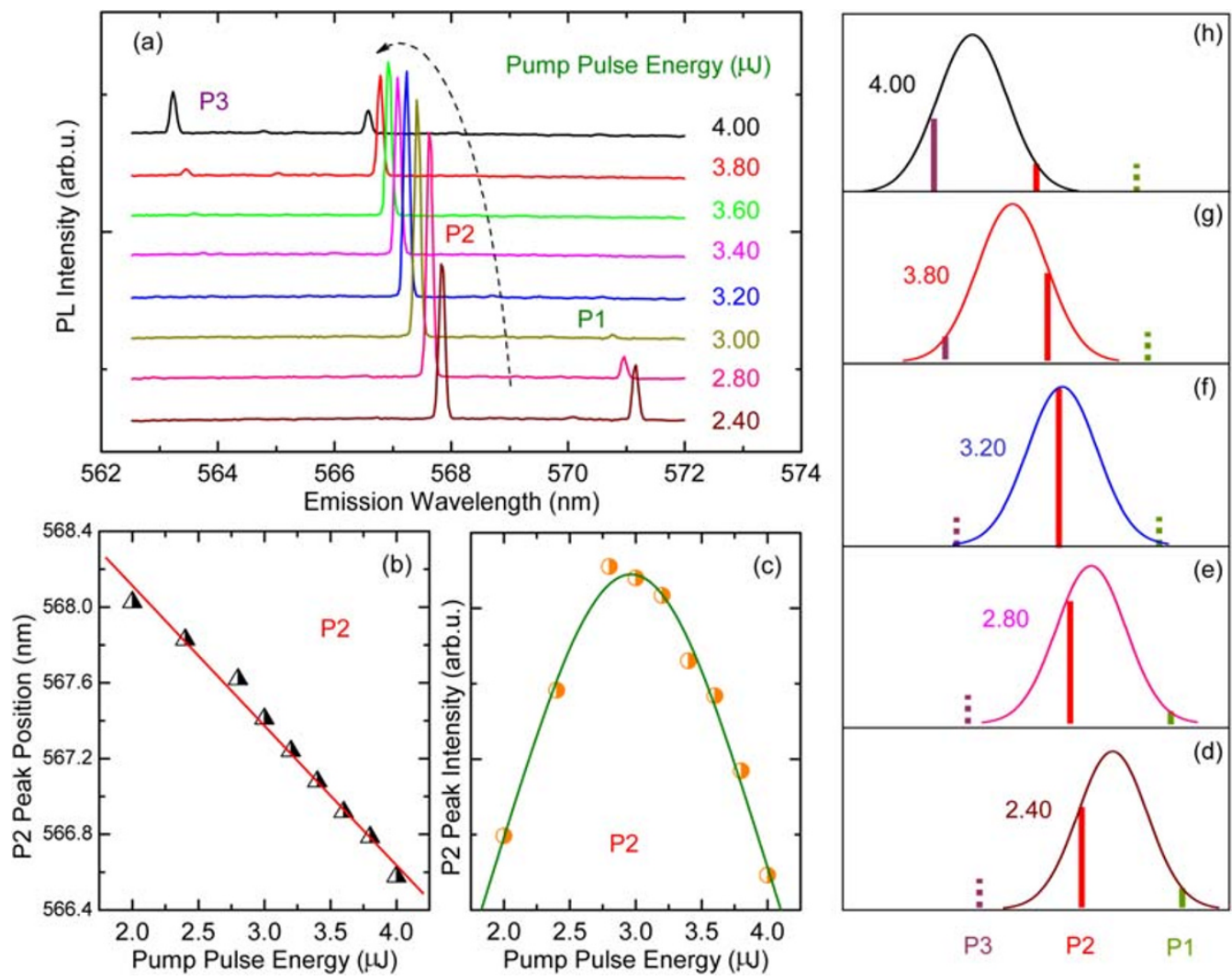

Figure $5 \mid$ Frequency shift and mode hopping. (a) Blue shift behavior of the hemisphere with diameter of $15 \mu \mathrm{m}$, where P1, P2, and P3 denote the three different peaks. (b) The evolution of P2 peak position with pump pulse energy. (c) The relationship between P2 peak intensity and pump pulse energy. (d)-(h) The schematic diagram shows the blue shift of optical gain (solid curves) and cavity mode (vertical lines). Solid lines denote the lasing action, while dashed lines mean no lasing occurred. The values given correspond to the pump pulse energy. 
experimental data. Moreover, the diameter $D$ is close to the value as shown in Figure 1e. Therefore, the lasing mechanism is ascribed to WGM.

Single longitudinal mode lasing. The absorption and emission of the dye molecules are plotted in the inset of Figure 4, where the black arrow denotes the pumping wavelength, and the patterned area represents the region where the optical gain larger than the loss can be achieved. As indicated in the inset of Figure 4, single mode lasing can be achieved if the mode spacing from the circular cavity is comparable to the width of the lasing region. Following this idea, we have indeed observed single mode lasing. The red curve in Figure 4 depicts the selected lasing emission from hemisphere with smaller diameter (Figure 1f, with diameter of $15 \mu \mathrm{m}$ ). The spectrum was captured under pump pulse energy of $3.4 \mu \mathrm{J}$ which is above the threshold. It can be seen that the lasing action is entirely occupied by a single longitudinal mode, which indicates the tunability of the proposed lasing structure. The $Q$ factor is calculated to be $\sim 4500$, which is smaller than the bigger hemisphere discussed above. This can be explained by the smaller hemisphere cavity volume, which provides less spatial overlap between the WGM and the dye molecules gain medium, resulting in more light leakage (optical loss) from the WGM cavity ${ }^{19,32}$.

It is very interesting to see the pump pulse energy dependence of the lasing emission from the small hemisphere, which was shown in
Figure 5a. All the curves are normalized and shifted vertically for better comparison. It can be seen clearly that the same hemisphere shows different lasing peaks under different pump pulse energies, which can be classified into different groups denoted as P1, P2 and P3, respectively. As depicted in Figure 5a, all the peaks show a gradual and nonreversible blue shift with increase pump pulse energy. In contrast, the evolution of the peak intensity is more complicated. For example, with the increase of pump pulse energy, P1 demonstrates a monotonous decrease and totally disappears when pump pulse energy higher than $3.20 \mu \mathrm{J}$, which is the reverse phenomenon compared to P3. More surprisingly, the change of P2 is nonlinear, which shows an increase and then decrease of the intensity. For better understanding, the peak position and peak intensity of P2 changed with pump pulse energy are plotted in Figure $5 \mathrm{~b}$ and $6 \mathrm{c}$, respectively. It can be seen that the peak position of $\mathrm{P} 2$ is monotonous dependent on the pump pulse energy, while the peak intensity shows a peak-like profile.

The change of lasing modes can be well-explained by frequency shift and mode hopping. Under high pulse laser excitation, the size of the hemisphere will slightly change and the radiation induced photooxidation will take place ${ }^{25,33}$. As is well-known, the lasing emission is very sensitive to the cavity length and the gain profile $\mathrm{e}^{34}$. However, if only the change of cavity size is responsible to the observation, P1 should show a continuous increase rather than decrease. On the other hand, if the observation is due to photo-oxidation only, then
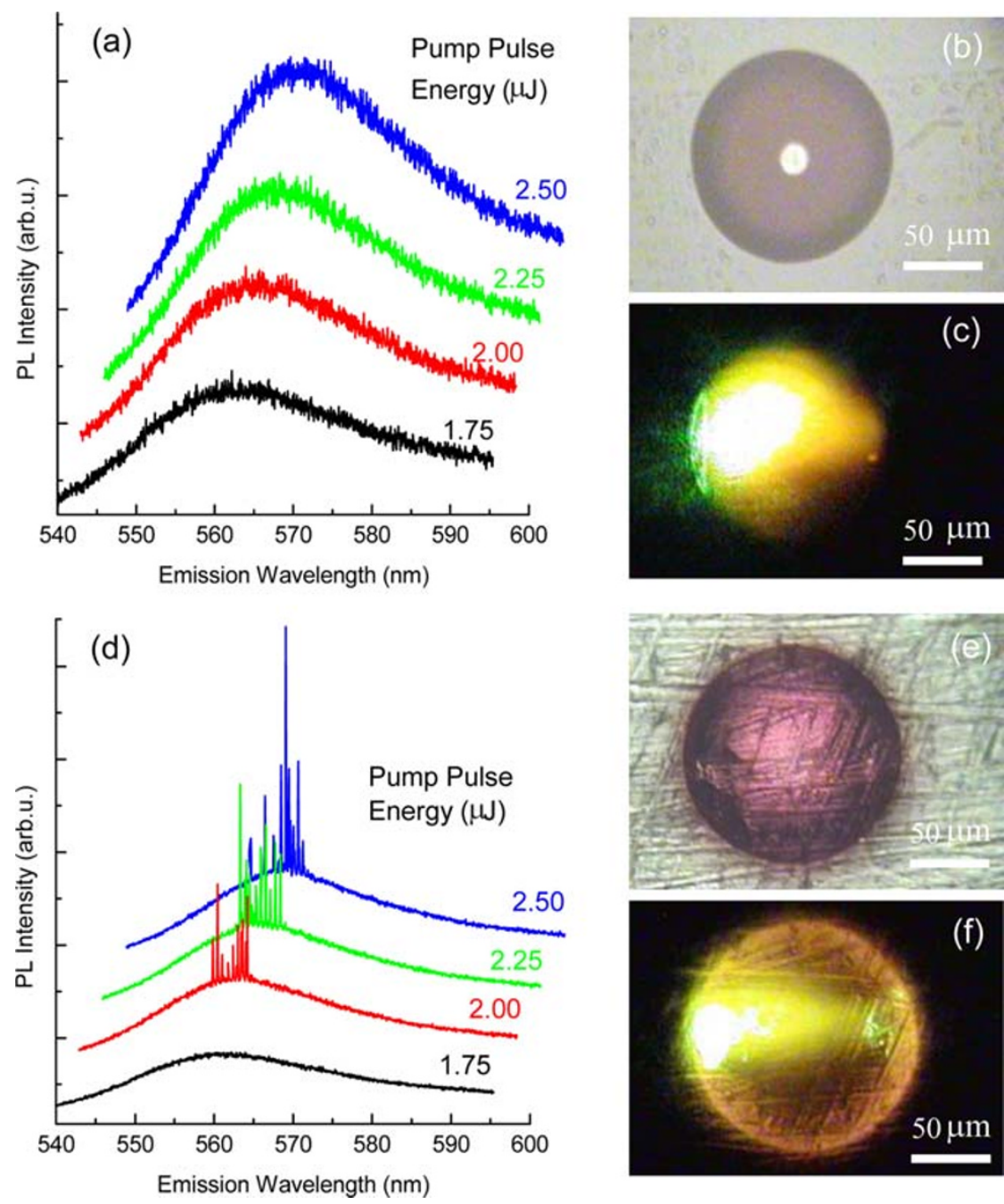

Figure 6 The importance of 3D confinement. (a) PL emission from microresonator on glass substrate. (b) Optical image and (c) PL image of the

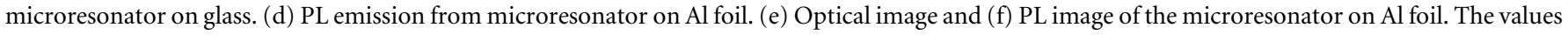
in (a) and (d) indicate the pump pulse energies for the measurement. 
all the peaks should keep the same position. In order to explain the frequency shift and mode hopping, schematic diagrams as drawn in Figure $5 \mathrm{~d}$ to $6 \mathrm{~h}$. The curves stand for the optical gain profile of the dye molecules, while the vertical solid/dashed lines represent the cavity mode of WGM resonators. As can be seen in Figure 5d, at low pump pulse energy $2.40 \mu \mathrm{J}$, only the $\mathrm{P} 1$ and $\mathrm{P} 2$ modes are covered inside the optical gain due to the small diameter of the hemisphere. In this situation, only two lasing peaks are able to be observed. With the increase of pump pulse energy, the laser pumping will introduce the slight change of cavity size and photo-oxidation. Therefore, the optical gain profile and the cavity mode will demonstrate a blue shift simultaneously. However, it is noted that the speed of the optical gain shifting is faster than the cavity mode, which results in single longitudinal mode lasing under pump pulse energy of $3.20 \mu \mathrm{J}$ as shown in Figure 5d. Followed this explanation, all the experimental data plotted in Figure $5 \mathrm{a}$ can be well-understood. Therefore, the information extracted from Figure $5 \mathrm{~b}$ reflects the change of cavity size with pump pulse energy. As a matter of fact, the modal blue shift is slower than the previous report ${ }^{25}$, which should be ascribed to the better protection of dye molecules inside epoxy resin. Moreover, the evolution of the P2 peak intensity roughly represents the optical gain profile of the dye molecules.

3D confinement. We also measure the emission from spherical microresonator on top of glass substrate. As shown in Figure 6(a), no lasing action observed under optical excitation. Moreover, the PL image given in Figure 6(c) is different from Figure 3, where no circular pattern can be observed. This control experiment implies the important role of the bottom planar DBR. Thanks to the bottom DBR and the higher refractive index of the epoxy resin compared to the surrounding environment (air), the light emitted from the dye molecules is totally confined inside the hemisphere, and propagates in the plane parallel to the substrate by multiple total internal refractions. The resonance condition is fulfilled once the light reaches the origin point with the same phase after one circulation. Therefore, the observation of high quality lasing was ascribed to the hybrid microresonator combined hemispherical resonator with planer DBR, which results in the $3 \mathrm{D}$ confinement. It is also possible to replace the bottom planer DBR by a metallic substrate, such as Aluminum (Al) foil. Although the surface of the $\mathrm{Al}$ foil is not very smooth, as shown in Figure 6(e), under the same measurement condition, lasing emission from spherical microresonator on top of $\mathrm{Al}$ foil can be observed, which further verifies the importance of high reflectivity of the bottom plane. This proof-of-concept demonstration may also provide a possible platform for investigation of interaction between surface plasma and optical microresonator, and also points an effective route of fabricating microlasers on flexible substrate.

\section{Discussion}

We demonstrate a feasible technique for hybrid self-assembled 3D confined hemispheres through hydrophobic effect for optical microresonators. At room temperature, multimode and singe-mode lasing were observed from dye-doped hemisphere with different sizes. The blue shift of lasing modes under different pump pulse energy was investigated carefully, and the results indicate both the slightly change of cavity size and the radiation induced photo-oxidation are responsible for the frequency shift and mode hopping. We believe an even lower threshold and stable performance of the microlasers can be obtained through better protection of the gain materials. It is important to note that the proposed microlaser could be pumped efficiently by a vertical cavity semiconductor laser, or even a high brightness light-emitting diode with a suitable operating wavelength. Owing to the simple, high $Q$ resonating property and $3 \mathrm{D}$ confinement, our system offers a versatile candidate for the future exploration of light/matter interaction as well as microlasers for photonic devices.

\section{Methods}

Optical measurement setup. A He-Cd laser with laser line of $325 \mathrm{~nm}$ was used as the $\mathrm{CW}$ excitation source. For high density excitation, the laser source was replaced by a frequency-doubled, Q-switched Nd:YAG laser which produced green pulse at the wavelength of $532 \mathrm{~nm}$. The pulse width and repetition rate of the laser are about $1 \mathrm{~ns}$ and $60 \mathrm{~Hz}$, respectively. The excitation laser was guided and focused to a spot of $1.5 \mathrm{~mm}$ in diameter at a grazing angle $\sim 45^{\circ}$ normal to the DBR substrate. The sample was put on an $\mathrm{X}-\mathrm{Y}-\mathrm{Z}$ movable stage. The signal emitted from individual hemisphere was collected with an objective $(50 \times$, numerical aperture $=0.42)$, and delivered to a camera for imaging, or focused into an optical fiber and directed into a $750 \mathrm{~mm}$ monochromator combined with a back-illuminated silicon charged coupled device (CCD) for spectral recording. The collection of the emission can be either from the top or the edge of the hemispheres. With the selected 600 grooves $/ \mathrm{mm}$ grating (blaze at $500 \mathrm{~nm}$ ) and input slit width of $20 \mu \mathrm{m}$, a spectral resolution of $\sim 0.05 \mathrm{~nm}$ can be achieved. Moreover, a shutter was used to block the pump beam at all times except during the $200 \mathrm{~ms}$ exposure periods of the CCD camera. And the pump power can be adjusted by a variable-density filter. Schematic diagram of the experimental setup can be found in Figure S3, Supplementary Information.

1. Vahala, K. J. Optical microcavities. Nature 424, 839 (2003).

2. Barrelet, C. J. et al. Hybrid single-nanowire photonic crystal and microresonator structures. Nano Lett. 6, 11 (2005).

3. Park, Y.-S., Cook, A. K. \& Wang, H. Cavity QED with diamond nanocrystals and silica microspheres. Nano Lett. 6, 2075 (2006).

4. Humar, M. \& Musevic, I. 3D microlasers from self-assembled cholesteric liquidcrystal microdroplets. Opt. Express 18, 26995 (2010).

5. Ning, C. Z. Semiconductor nanolasers. Phys. Status Solidi B 247, 774 (2010).

6. Gibbs, H. M., Khitrova, G. \& Koch, S. W. Exciton-polariton light-semiconductor coupling effects. Nat. Photonics 5, 273 (2011).

7. Reithmaier, J. P. et al. Strong coupling in a single quantum dot-semiconductor microcavity system. Nature 432, 197 (2004).

8. Humar, M., Ravnik, M., Pajk, S. \& Musevic, I. Electrically tunable liquid crystal optical microresonators. Nat. Photonics 3, 595 (2009).

9. Vollmer, F. \& Arnold, S. Whispering-gallery-mode biosensing: label-free detection down to single molecules. Nat. Methods 5, 591 (2008).

10. Someya, T. et al. Room temperature lasing at blue wavelengths in gallium nitride microcavities. Science 285, 1905 (1999).

11. Chen, R., Sun, H. D., Wang, T., Hui, K. N. \& Choi, H. W. Optically pumped ultraviolet lasing from nitride nanopillars at room temperature. Appl. Phys. Lett. 96, 241101 (2010).

12. Strauf, S. et al. Self-tuned quantum dot gain in photonic crystal lasers. Phys. Rev. Lett. 96, 127404 (2006).

13. Painter, O. et al. Two-dimensional photonic band-gap defect mode laser. Science 284, 1819 (1999).

14. Cao, H. et al. Random laser action in semiconductor powder. Phys. Rev. Lett. 82, 2278 (1999).

15. Chen, R. et al. Excitonic properties and near-infrared coherent random lasing in vertically aligned CdSe nanowires. Adv. Mater. 23, 1404 (2011).

16. Nezhad, M. P. et al. Room-temperature subwavelength metallo-dielectric lasers. Nat. Photonics 4, 395 (2010).

17. Ma, R. M., Oulton, R. F., Sorger, V. J., Bartal, G. \& Zhang, X. Room-temperature sub-diffraction-limited plasmon laser by total internal reflection. Nat. Mater. 10, 110 (2011).

18. Samuel, I. D. W. \& Turnbull, G. A. Organic semiconductor lasers. Chem. Rev. 107, 1272 (2007).

19. Chen, R., Ling, B., Sun, X. W. \& Sun, H. D. Room temperature excitonic whispering gallery mode lasing from high-quality hexagonal $\mathrm{ZnO}$ microdisks. Adv. Mater. 23, 2199 (2011).

20. Yim, T. J., Zentgraf, T., Min, B. \& Zhang, X. All-liquid photonic microcavity stabilized by quantum dots. J. Am. Chem. Soc. 132, 2154 (2010).

21. Lee, W. et al. A quasi-droplet optofluidic ring resonator laser using a microbubble. Appl. Phys. Lett. 99, 091102 (2011).

22. Cai, M., Painter, O., Vahala, K. J. \& Sercel, P. C. Fiber-coupled microsphere laser. Optics Letters 25, 1430 (2000).

23. Snee, P. T., Chan, Y., Nocera, D. G. \& Bawendi, M. G. Whispering-gallery-mode lasing from a semiconductor nanocrystal/microsphere resonator composite. $A d v$. Mater. 17, 1131 (2005).

24. Reitzenstein, S. \& Forchel, A. Quantum dot micropillars. J. Phys. D: Appl. Phys. 43, 033001 (2010).

25. Schäfer, J. et al. Quantum dot microdrop laser. Nano Lett. 8, 1709 (2008).

26. Qian, S. X., Snow, J. B., Tzeng, H. M. \& Chang, R. K. Lasing droplets - highlighting the liquid-air interface by laser-emission. Science 231, 486 (1986).

27. Kiraz, A. et al. Lasing from single, stationary, dye-doped glycerol/water microdroplets located on a superhydrophobic surface. Opt. Commun. 276, 145 (2007).

28. Haase, J. et al. Hemispherical resonators with embedded nanocrystal quantum rod emitters. Appl. Phys. Lett. 97, 211101 (2010) 
29. Xiao, Y. et al. Single-nanowire single-mode laser. Nano Lett. 11, 1122 (2011).

30. Ta, V. D., Chen, R. \& Sun, H. D. Self-assembled flexible microlasers. Adv. Mater. DOI: 10.1002/adma.201103409.

31. Bergman, L., Chen, X.-B., Morrison, J. L., Huso, J. \& Purdy, A. P. Photoluminescence dynamics in ensembles of wide-band-gap nanocrystallites and powders. J. Appl. Phys. 96, 675 (2004).

32. Gargas, D. J. et al. Whispering gallery mode lasing from zinc oxide hexagonal nanodisks. ACS Nano 4, 3270 (2010).

33. Nazzal, A. Y. et al. Environmental effects on photoluminescence of highly luminescent $\mathrm{CdSe}$ and $\mathrm{CdSe} / \mathrm{ZnS}$ core/shell nanocrystals in polymer thin films. J. Phys. Chem. B 108, 5507 (2004).

34. Moon, H. J. et al. Cavity-Q-driven spectral shift in a cylindrical whisperinggallery-mode microcavity laser. Appl. Phys. Lett. 76, 3679 (2000).

\section{Acknowledgements}

Support from the Singapore Ministry of Education through the Academic Research Fund (Tier 1) under Project No. RG63/10 and from the Singapore National Research Foundation through the Competitive Research Programme (CRP) under Project No. NRF-CRP6-2010-02 is gratefully acknowledged.

\section{Author Contribution}

H. D. S. proposed and guided the overall project. R.C. and V.D.T. performed all experiments and analyzed the results. All authors discussed the results and substantially contributed to the manuscript.

\section{Additional information}

Supplementary information accompanies this paper at http://www.nature.com/ scientificreports

Competing financial interests: The authors declare no competing financial interests.

License: This work is licensed under a Creative Commons

Attribution-NonCommercial-ShareAlike 3.0 Unported License. To view a copy of this license, visit http://creativecommons.org/licenses/by-nc-sa/3.0/

How to cite this article: Chen, R., Ta, V.D. \& Sun, H.D. Single Mode Lasing from Hybrid Hemispherical Microresonators. Sci. Rep. 2, 244; DOI:10.1038/srep00244 (2012). 